\title{
Biliary Complications Following Liver Transplantation in the Model for End-Stage Liver Disease Era: Effect of Donor, Recipient, and Technical Factors
}

\author{
Theodore H. Welling, David G. Heidt, Michael J. Englesbe, John C. Magee, Randall S. Sung, \\ Darrell A. Campbell, Jeffrey D. Punch, and Shawn J. Pelletier \\ Division of Transplantation, University of Michigan Medical Center, Ann Arbor, MI
}

\begin{abstract}
Biliary complications remain a significant problem following liver transplantation in the Model for End-Stage Liver Disease (MELD) era. We hypothesized that donor, recipient, and technical variables may differentially affect anastomotic biliary complications in MELD era liver transplants. We reviewed 256 deceased donor liver transplants after the institution of MELD at our center and evaluated these variables' association with anastomotic biliary complications. The bile leak rate was $18 \%$, and the stricture rate was $23 \%$. Univariate analysis revealed that recipient age, MELD, donor age, and warm ischemia were risk factors for leak, whereas a Roux limb or stent was protective. A bile leak was a risk factor for anastomotic stricture, whereas use of histidine tryptophan ketoglutarate (HTK) versus University of Wisconsin (UW) solution was protective. Additionally, use of a transcystic tube/stent was also protective. Multivariate analysis showed that warm ischemia was the only independent risk factor for a leak, whereas development of a leak was the only independent risk factor for a stricture. HTK versus UW use and transcystic tube/stent use were the only independent protective factors against stricture. Use of an internal stent trended in the multivariate analysis toward being protective against leaks and strictures, but this was not quite statistically significant. This represents one of the first MELD era studies of deceased donor liver transplants evaluating factors affecting the incidence of anastomotic bile leaks and strictures. Donor, recipient, and technical factors appear to differentially affect the incidence of anastomotic biliary complications, with warm ischemia, use of HTK, and use of a stent emerging as the most important variables. Liver Transpl 14:73-80, 2008. $\odot 2007$ AASLD.
\end{abstract}

Received March 22, 2007; accepted August 17, 2007.

Although the indications and overall success rate for liver transplantation have increased steadily over the past couple of decades, biliary complications continue to add morbidity to this procedure. The majority of these complications are related to the biliary anastomosis, with the earliest series noting biliary complication rates of $10 \%-30 \%{ }^{1-5}$ Despite advances in surgical technique, biliary complication rates remain high in contemporary series. ${ }^{6}$ A variety of interventions are available to treat and diagnose biliary complications, but these complications still represent a large source of morbidity and financial burden to liver transplant patients. $^{7}$

Multiple risk factors for biliary complications have been identified. Use of living donor livers is associated with a higher risk of biliary complications. ${ }^{6,8}$ Placement of a T-tube has also been identified as a culprit in the development of these complications. ${ }^{9}$ Clearly, bile leaks are thought to promote development of biliary strictures, ${ }^{1,6}$ and hepatic arterial complications promote both bile leaks and strictures. ${ }^{1,10,11}$ Debate has centered on the type of biliary reconstruction, ${ }^{1,12,13}$ use of

Abbreviations: CI, confidence interval; Cr, creatinine; HAT, hepatic artery thrombosis; HTK, histidine tryptophan ketoglutarate; MELD, Model for End-Stage Liver Disease; NA, not applicable; OR, odds ratio; PBC, primary biliary cirrhosis; PNF, primary nonfunction; PSC, primary sclerosing cholangitis; SD, standard deviation; UW, University of Wisconsin.

Address reprint requests to Theodore H. Welling, M.D., 1500 East Medical Center Drive, 2926 Taubman Center, Ann Arbor, MI 48109. Telephone: 734-936-8363; FAX: 734-763-3187; E-mail: twelling @umich.edu

DOI 10.1002/lt.21354

Published online in Wiley InterScience (www.interscience.wiley.com). 
stents, ${ }^{12,14}$ and recipient factors of illness severity, such as preoperative serum bilirubin and international normalized ratio. ${ }^{6}$ However, the relative influence of these factors and their interactions with other variables on the risk of biliary complications is not completely known in contemporary liver transplant experience.

Since February 27, 2002, the Model for End-Stage Liver Disease (MELD) allocation system has shifted liver transplant allocation priority toward sicker patients, ${ }^{15}$ while at the same time donor criteria have expanded in an attempt to keep up with the growing list of liver transplant candidates. In addition, overall indications for transplantation and immunosuppressive regimens have changed over time. MELD scores and MELD components clearly affect survival and complication rates, specifically in certain patient populations. ${ }^{16,17}$ The overall impact of these changes in liver transplantation policy on the incidence of biliary complications among decease donor liver transplants has not been described. In addition, a contemporary detailed analysis of donor, recipient, and technical issues that may contribute to or be protective against these morbid complications has yet to be performed for the MELD era. We therefore examined the influence of these variables on the incidence of anastomotic biliary complications during the MELD era and hypothesized that they differentially impact the development of anastomotic bile leaks and strictures. To test this hypothesis, we retrospectively assessed the association of donor, recipient, and technical variables with biliary anastomotic complications in 256 consecutive deceased donor liver transplants following institution of the MELD allocation policy at our center.

\section{PATIENTS AND METHODS}

\section{Study Design}

A total of 256 consecutive deceased donor liver transplants among 238 patients were reviewed at the University of Michigan Medical Center between July 1, 2002 and June 30, 2005. Patients were identified by an administrative database, and all data were retrieved from patient records where appropriate. Procurement records were obtained from the Michigan Organ Procurement Organization (Gift of Life Michigan). All patients had at least 6 months of follow-up. To capture the effects of as many potential variables as possible on biliary complications, all transplants were evaluated regardless of age, etiology of liver failure/transplant indication, or method of biliary reconstruction. All data for the current study were collected in accordance with the University of Michigan Institutional Review Board.

Endoscopic retrograde cholangiopancreatogram, percutaneous transhepatic cholangiogram, or transcystic tube injections were performed for clinical suspicion of a bile leak or stricture where appropriate. A transcystic tube was placed selectively in some patients undergoing a choledochocholedochostomy at the discretion of the surgeon's practice preference. Briefly, this tube served as a combined external and internal stent that traversed the duct-to-duct anastomosis and exited via the cystic duct and gallbladder fossa. A minimum of 1 cholangiogram was performed usually within the first 10 postoperative days in patients receiving a transcystic tube, which was discontinued at 6 weeks postoperatively. Additional cholangiograms were performed via the transcystic tube as clinically indicated. An internal stent alone was also used in selected cases, again at the discretion of the surgeon. A Roux-en-Y hepaticojejunostomy was used at the discretion of the surgeon, usually in patients with primary sclerosing cholangitis (PSC) or biliary atresia. Otherwise, patients underwent an end-to-end choledochocholedochostomy. Anastomoses were created with absorbable sutures in an interrupted fashion. Patients were classified as having an anastomotic bile leak if they had evidence of a leak from endoscopic retrograde cholangiopancreatogram, percutaneous transhepatic cholangiogram, or transcystic tube cholangiograms. In a few patients, a bile leak was diagnosed upon re-exploration for either suspected thrombosis or bowel perforation. An anastomotic biliary stricture was defined as any obstructive lesion at the anastomosis detected postoperatively by cholangiography that required a therapeutic intervention, such as stent placement, cholangioplasty, or stent exchange. A stricture was not defined as a size mismatch. Intrahepatic (nonanastomotic) strictures were not included in this analysis.

The immunosuppression regimen usually consisted of triple therapy and included either cyclosporine or tacrolimus with prednisone and mycophenolate mofetil. A separate analysis of 50 patients who were part of a steroid avoidance protocol showed no effect of this protocol on the incidence of biliary complications, and these recipients were included in the present analysis. ${ }^{18}$ Immunosuppression was decreased or minimized as appropriate in the presence of significant infections or adverse side effects.

University of Wisconsin (UW) solution was used as a preservative during organ procurement during the first half of the study period. Subsequently, a decision was made by the local organ procurement organization to use histidine tryptophan ketoglutarate (HTK) preservation solution for the remainder of the period. All donors were deceased; no donation after cardiac death donors were included in the analysis. Donor procurement was performed via a retrograde aortic flush with a supraceliac aortic cross clamp. Blood and perfusate were vented through the cavoatrial junction or the infrarenal inferior vena cava. Approximately 4-5 L of either HTK or UW solution was used, and this was followed by packing in the same solution. The gallbladder and bile duct were flushed on the back table. A portal venous flush of 250-350 mL was routinely performed on the back table.

\section{Statistical Analysis}

Categorical variables were expressed as a percentage, and continuous variables were expressed as a mean \pm standard deviation. A chi-square or unpaired $t$ test was used where appropriate. Univariate logistic regression 
analysis was performed on the following variables: donor age, donor gender, donor creatinine, recipient age, recipient gender, recipient match $M E L D$, recipient diagnosis/indication, warm ischemia time, cold ischemia time, total ischemia time, use of HTK versus UW preservation solution, use of an internal stent, use of a transcystic tube, and use of a Roux-en-Y choledochojejunostomy. A multivariate logistic regression analysis was performed on variables found to be statistically significant in the univariate analysis or with $P<0.20$ and on variables that had other potential biologic or clinical relevance.

All statistical analyses were performed with SAS software (Statview 5.0.1, SAS Institute, Inc., Cary, NC). Variables in all analyses with a $P$ value of less than 0.05 were considered significant.

\section{RESULTS}

\section{Demographics and Clinical Outcomes}

The baseline recipient, donor, and technical characteristics of the 256 transplants for the MELD era $(7 / 02$ $6 / 05)$ are listed in Table 1 . The mean age of recipients was 43 years, with a majority being male (63\%). A majority of these recipients were classified as adults $(83 \%$, age 17 or greater). Of the children, 31 were small (age $<$ 12), with 12 being adolescent (age $>12$ ). The mean MELD/Pediatric End-Stage Liver Disease score was $22.8 \pm 6$. Hepatitis $\mathrm{C}$ was the most common indication for transplantation, making up $37 \%$ of the recipients. Biliary atresia was the most common indication for small children ( $\mathrm{n}=15$ or $48 \%$ ). PSC was the most common cholestatic indication for transplantation, making up $6.6 \%$ of the recipients.

The mean donor age, as expected, was lower than the recipient age and was 34 years. A majority of donors were male, comprising 60\%. The proportions of donors for whom HTK and UW solutions were used were 55\% and $45 \%$, respectively. A choledochocholedochostomy was performed in most cases, with $23 \%$ undergoing a Roux-en-Y hepaticojejunostomy. An internal stent was used in $14 \%$ of transplants, whereas a transcystic tube/stent (internal/external) was used in 16\%. A Roux limb was used in all children with biliary atresia, whereas 9 of 16 small children without biliary atresia underwent Roux limb reconstruction.

Following liver transplantation, there were 41 deaths (16\%), 3 hepatic artery thromboses, and 3 cases of primary nonfunction (Table 2). A cholangiogram was performed for clinical indications or for surveillance of a transcystic tube in 53\% of transplants. Essentially, all those with a transcystic tube were studied, whereas $44 \%$ of those without a tube underwent a cholangiogram on the basis of clinical indications. An anastomotic bile leak or anastomotic stricture was diagnosed in $18 \%$ and $23 \%$ of transplants, respectively. For pediatric patients, there were a total of 2 anastomotic bile leaks, 1 in an infant with biliary atresia and 1 in an adolescent with fulminant hepatic failure. Of those that had a leak,

\begin{tabular}{|c|c|}
\hline \multicolumn{2}{|c|}{ Recipient characteristics, n (\%) } \\
\hline Age (years), mean ( $\pm \mathrm{SD})$ & 43 (19) \\
\hline Female & 89 (37) \\
\hline Male & $153(63)$ \\
\hline Pediatric (age < 17) & 43 (17) \\
\hline Adult & $213(83)$ \\
\hline MELD, mean $( \pm \mathrm{SD})$ & $22.8(6)$ \\
\hline \multicolumn{2}{|l|}{ Etiology or indication, $\mathrm{n}(\%)$} \\
\hline Hepatitis C & $95(37)$ \\
\hline Alcohol or cryptogenic & $62(24)$ \\
\hline PSC & $17(6.6)$ \\
\hline Fulminant & $14(5.5)$ \\
\hline Retransplant & $15(5.9)$ \\
\hline $\mathrm{PNF} / \mathrm{HAT}$ & $6(2.3)$ \\
\hline $\mathrm{PBC}$ & $10(3.9)$ \\
\hline Biliary atresia & $16(6.3)$ \\
\hline Hepatitis B & $8(3.1)$ \\
\hline Autoimmune & $10(6.3)$ \\
\hline Other & 4 (1.6) \\
\hline \multicolumn{2}{|l|}{ Donor characteristics, n (\%) } \\
\hline Age (years), mean ( \pm SD) & $34(8)$ \\
\hline Female & $83(40)$ \\
\hline Male & $123(60)$ \\
\hline Pediatric (age < 17) & 40 (19) \\
\hline Adult & $166(81)$ \\
\hline \multicolumn{2}{|l|}{ Preservation, n (\%) } \\
\hline HTK solution & $141(55)$ \\
\hline UW solution & $115(45)$ \\
\hline \multicolumn{2}{|l|}{ Biliary reconstruction, $\mathrm{n}(\%)$} \\
\hline Roux limb & $58(23)$ \\
\hline Internal stent & 37 (14) \\
\hline Transcystic tube & $42(16)$ \\
\hline No stent/tube/Roux & $119(46)$ \\
\hline $\begin{array}{l}\text { Abbreviations: HAT, hepatic } \\
\text { histidine tryptophan ketoglutar } \\
\text { Stage Liver Disease; PBC, prim } \\
\text { primary nonfunction; PSC, prim } \\
\text { SD, standard deviation; UW, Un }\end{array}$ & $\begin{array}{l}\text { nbosis; HTK, } \\
\text { Iodel for End- } \\
\text { irrhosis; PNF, } \\
\text { g cholangitis; } \\
\text { isconsin. }\end{array}$ \\
\hline
\end{tabular}

\begin{tabular}{|lr|}
\hline \multicolumn{2}{|c|}{ TABLE 2. Clinical Outcomes [n (\%)] } \\
\hline Mortality & $41(16)$ \\
PNF & $3(1.2)$ \\
HAT & $3(1.2)$ \\
Cholangiogram & $136(53)$ \\
Anastomotic bile leak & $47(18)$ \\
Anastomotic stricture & $59(23)$ \\
Leak and subsequent stricture & $21(8.2)$ \\
\hline Abbreviations: HAT, hepatic artery thrombosis; PNF, \\
primary nonfunction.
\end{tabular}

$44.7 \%$ subsequently developed an anastomotic stricture, which comprised $8.2 \%$ of the total transplants. The incidence of death did not appear to be associated with the development of a bile leak [odds ratio (OR) 0.78, confidence interval (CI) 0.31-1.99, $P=$ 0.603] or a stricture (OR 0.70, CI 0.29-1.68, $P=$ $0.421)$. 


\begin{tabular}{|c|c|c|c|}
\hline & No Leak & Bile Leak & $P$ Value \\
\hline Recipient age (years), mean ( $\pm \mathrm{SD})$ & $40.8(11)$ & $50.4(20)$ & 0.003 \\
\hline Recipient gender (male versus female, \%) & 62.8 & 65.1 & 0.081 \\
\hline MELD, mean ( $\pm \mathrm{SD})$ & $22.3(6)$ & $24.6(7)$ & 0.021 \\
\hline Donor age (years), mean ( \pm SD) & $32.0(18)$ & $41.8(16)$ & 0.003 \\
\hline Donor gender (male versus female, \%) & 59.2 & 57.5 & 0.663 \\
\hline Donor $\mathrm{Cr}$, mean $( \pm \mathrm{SD})$ & $1.0(0.8)$ & $1.2(1.5)$ & 0.365 \\
\hline Preservation (HTK versus UW, \%) & 56.5 & 48.9 & 0.349 \\
\hline Warm ischemia (minutes), mean $( \pm \mathrm{SD})$ & $41.9(16)$ & $49.1(23)$ & 0.024 \\
\hline Cold ischemia (minutes), mean ( $\pm \mathrm{SD}$ ) & $522(154)$ & $488(98)$ & 0.182 \\
\hline Total ischemia (minutes), mean ( $\pm \mathrm{SD})$ & $564(154)$ & $537(100)$ & 0.298 \\
\hline
\end{tabular}

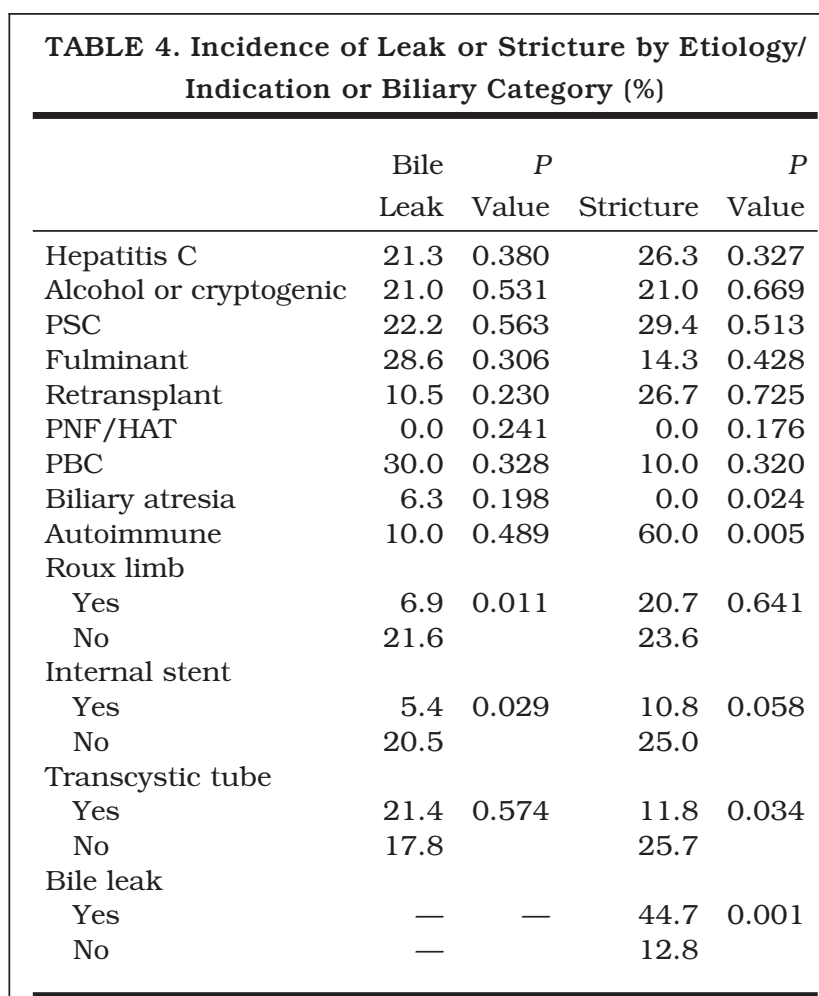

Abbreviations: HAT, hepatic artery thrombosis; PBC, primary biliary cirrhosis; PNF, primary nonfunction; PSC, primary sclerosing cholangitis.

\section{Predictors of Anastomotic Bile Leak}

The distribution of bile leaks among donor, recipient, and technical variables is listed in Tables 3 and 4 . The mean recipient age was higher for those who experienced a bile leak (50.4 versus 40.8 years, $P=0.003$ ) than for those without a leak. In addition, the mean MELD score was significantly higher in those that leaked (24.6 versus 22.3, $P=0.021$ ). Recipient gender and disease etiology were not significantly associated with a bile leak, although the sample size for some of these disease categories was small. There were no pa- tients with primary nonfunction or hepatic artery thrombosis that experienced a bile leak, only 1 of 10 patients with autoimmune hepatitis had a leak, and only 1 of 16 patients with biliary atresia had a leak. Donor age was also significantly higher in cases that had a bile leak (41.8 versus 32.0, $P=0.003$ ). Donor gender, creatinine, and preservation solution were not associated with an increased risk of bile leak. Warm ischemia time was significantly prolonged in cases with a bile leak (49.1 versus 41.9 minutes, $P=0.024$ ), whereas cold and total ischemia times were not significantly different. The use of a Roux limb or an internal stent reduced the leak rate to $6.9 \%(P=0.011)$ and $5.4 \%(P=0.029)$ of cases, respectively. A transcystic tube did not appear to reduce the incidence of a bile leak, with an incidence of $21.4 \%$.

Univariate analysis of these risk factors using logistic regression (Table 5) showed that recipient age, donor age, MELD score, and warm ischemia time were all positive predictors for the development of an anastomotic bile leak. However, use of a Roux limb or an internal stent was protective against the development of a bile leak, with ORs of 0.27 (CI 0.09-0.78) and 0.22 (CI 0.05-0.96), respectively. Variables found to be significant in the univariate analysis or with a $P$ value of less than 0.20 or having additional clinical importance were analyzed in a multivariate fashion (Table 6). Warm ischemia time appeared to be the only variable independently associated with the development of a bile leak $(P=0.044)$. There was a trend for an increased risk of a bile leak with increasing MELD in the multivariate analysis, but this did not meet statistical significance $(P=0.085)$. Use of a Roux limb or an internal stent was not independently protective in the multivariate analysis $(P=0.149$ and $P=0.072$, respectively).

\section{Predictors of Anastomotic Biliary Stricture}

The same variables evaluated for the distribution of bile leaks were analyzed for the distribution of anastomotic strictures (Tables 4 and 7). In contrast to bile leaks, the mean recipient age and mean MELD score did not differ significantly between cases in which a stricture oc- 


\begin{tabular}{|c|c|c|c|c|}
\hline & \multicolumn{2}{|r|}{ Bile Leak } & \multicolumn{2}{|r|}{ Stricture } \\
\hline & OR (CI) & $P$ Value & OR (CI) & $P$ Value \\
\hline Recipient age & $1.03(1.01-1.06)$ & 0.005 & $1.01(1.00-1.03)$ & 0.151 \\
\hline Recipient gender (male versus female) & $1.05(0.53-2.09)$ & 0.890 & $1.58(0.83-3.03)$ & 0.166 \\
\hline MELD & $1.06(1.01-1.12)$ & 0.023 & $0.99(0.94-1.04)$ & 0.712 \\
\hline \multicolumn{5}{|l|}{ Etiology/indication } \\
\hline Hepatitis C & $0.75(0.39-1.43)$ & 0.381 & $0.74(0.41-1.35)$ & 0.328 \\
\hline Alcohol or cryptogenic & $0.80(0.39-1.63)$ & 0.532 & $1.16(0.58-2.33)$ & 0.669 \\
\hline PSC & $1.41(0.44-4.53)$ & 0.565 & $1.44(0.48-4.25)$ & 0.515 \\
\hline Fulminant & $0.54(0.16-1.79)$ & 0.313 & $1.84(0.40-8.46)$ & 0.434 \\
\hline Retransplant & $0.30(0.04-2.37)$ & 0.256 & $1.24(0.38-4.04)$ & 0.725 \\
\hline Donor age & $1.03(1.01-1.05)$ & 0.003 & $1.02(1.00-1.04)$ & 0.054 \\
\hline Donor gender (male versus female) & $1.00(0.50-2.00)$ & 0.994 & $1.15(0.61-2.14)$ & 0.669 \\
\hline Donor $\mathrm{Cr}$ & $1.15(0.84-1.57)$ & 0.378 & $1.06(0.78-1.44)$ & 0.728 \\
\hline Preservation (HTK versus UW) & $0.74(0.39-1.39)$ & 0.350 & $0.48(0.26-0.86)$ & 0.014 \\
\hline Warm ischemia & $1.02(1.01-1.04)$ & 0.030 & $1.00(0.98-1.02)$ & 0.911 \\
\hline Cold ischemia & $1.00(0.99-1.00)$ & 0.183 & $1.00(0.99-1.00)$ & 0.999 \\
\hline Total ischemia & $1.00(0.99-1.00)$ & 0.298 & $1.00(0.99-1.00)$ & 0.998 \\
\hline Roux limb & $0.27(0.09-0.78)$ & 0.016 & $0.84(0.41-1.72)$ & 0.641 \\
\hline Internal stent & $0.22(0.05-0.96)$ & 0.044 & $0.36(0.12-1.07)$ & 0.067 \\
\hline Transcystic tube & $1.11(0.51-2.42)$ & 0.785 & $0.39(0.16-0.95)$ & 0.039 \\
\hline Bile leak & NA & NA & $3.78(1.92-7.45)$ & 0.0001 \\
\hline
\end{tabular}

Abbreviations: CI, confidence interval; Cr, creatinine; HTK, histidine tryptophan ketoglutarate; MELD, Model for End-Stage Liver Disease; NA, not applicable; OR, odds ratio; PSC, primary sclerosing cholangitis; UW, University of Wisconsin.

\begin{tabular}{|c|c|c|}
\hline \multicolumn{3}{|c|}{$\begin{array}{l}\text { TABLE 6. Multivariate Analysis of Variables for } \\
\text { Biliary Complications }\end{array}$} \\
\hline & OR (CI) & $P$ Value \\
\hline \multicolumn{3}{|l|}{ Bile leak } \\
\hline Recipient age & $1.02(0.99-1.05)$ & 0.179 \\
\hline MELD & $1.06(0.99-1.13)$ & 0.085 \\
\hline Donor age & $1.02(1.00-1.04)$ & 0.108 \\
\hline Warm ischemia & $1.02(1.00-1.04)$ & 0.044 \\
\hline Cold ischemia & $1.00(0.99-1.00)$ & 0.495 \\
\hline Roux limb & $0.44(0.15-1.34)$ & 0.149 \\
\hline Internal stent & $0.15(0.02-1.18)$ & 0.072 \\
\hline \multicolumn{3}{|l|}{ Stricture } \\
\hline Recipient age & $1.01(0.98-1.03)$ & 0.765 \\
\hline Donor age & $1.01(0.99-1.03)$ & 0.197 \\
\hline $\begin{array}{l}\text { Preservation } \\
\text { (HTK versus UW) }\end{array}$ & $0.40(0.21-0.75)$ & 0.005 \\
\hline Roux limb & $1.20(0.55-2.62)$ & 0.655 \\
\hline Internal stent & $0.37(0.12-1.16)$ & 0.088 \\
\hline Transcystic tube & $0.30(0.11-0.79)$ & 0.015 \\
\hline Bile leak & $3.63(1.77-7.46)$ & 0.001 \\
\hline
\end{tabular}

curred or did not. However, the mean donor age was significantly increased (38.2 versus $32.5, P=0.046$ ) for those with an anastomotic stricture. The incidence of a stricture was not significantly elevated among male recipients versus female recipients or for any particular disease etiology, with the exception of autoimmune hepatitis, which had a $60 \%$ incidence $(P=0.005)$. However, the incidence was significantly lower for patients with biliary atresia, with zero patients being affected $(P=0.024)$. However, there was a very small sample size for these 2 diagnoses. Use of HTK instead of UW preservation solution was associated with a lower incidence of anastomotic stricture, with $17.0 \%$ for HTK and $30.2 \%$ for UW $(P=0.023)$. Use of an internal stent was not quite significant with respect to the incidence of a stricture (10.8\%, $P=0.058)$. Use of a Roux limb carried an incidence of $20.7 \%$ for a stricture and was not significantly different $(P=0.641)$. However, a transcystic tube was significantly associated with a lower rate of stricture at $11.8 \%(P=0.034)$. Mean ischemia times and donor creatinine did not differ among cases with or without a stricture. Not surprisingly, development of a bile leak was associated with a $44.7 \%$ incidence of a subsequent stricture $(P=0.001)$.

The univariate analysis using logistic regression of variables that may be associated with the development of a stricture is presented in Table 5. Use of HTK appeared to be protective against a stricture (OR 0.48, CI 0.26-0.86, $P=0.014$ ), whereas use of an internal stent trended toward being protective but was not quite statistically significant (OR 0.36, CI 0.12-1.07, $P=0.067$ ). A transcystic tube was protective (OR 0.39, CI 0.16$0.95, P=0.039$ ). Donor age did not quite meet significance as a risk factor for stricture (OR 1.02, CI 1.001.04, $P=0.054)$. The development of a bile leak appeared to carry the highest relative risk among nominal variables for the development of an anastomotic stricture (OR 3.78, CI 1.92-7.45, $P=0.0001$ ). A multi- 


\begin{tabular}{|c|c|c|c|}
\hline & No Stricture & Stricture & $P$ Value \\
\hline Recipient age (years), mean ( $\pm \mathrm{SD})$ & $42.3(21)$ & $46.7(15)$ & 0.137 \\
\hline Recipient gender (male versus female, \%) & 61.8 & 71.9 & 0.164 \\
\hline MELD, mean $( \pm \mathrm{SD})$ & $22.7(6)$ & $22.5(6)$ & 0.842 \\
\hline Donor age (years), mean $( \pm \mathrm{SD})$ & $32.5(18)$ & $38.2(17)$ & 0.046 \\
\hline Donor gender (male versus female, \%) & 57.5 & 60.0 & 0.921 \\
\hline Donor $\mathrm{Cr}$, mean $( \pm \mathrm{SD})$ & $1.0(1.0)$ & $1.1(0.8)$ & 0.728 \\
\hline Preservation (HTK versus UW, \%) & 81.6 & 17.0 & 0.023 \\
\hline Warm ischemia (minutes), mean $( \pm S D)$ & $43.3(18)$ & $43.1(19)$ & 0.922 \\
\hline Cold ischemia (minutes), mean $( \pm \mathrm{SD})$ & $515(140)$ & $515(161)$ & 0.979 \\
\hline Total ischemia (minutes), mean ( $\pm \mathrm{SD})$ & $558(140)$ & $558(160)$ & 0.998 \\
\hline
\end{tabular}

variate analysis was subsequently performed (Table 6) and showed that use of HTK and use of a transcystic tube were independently protective against the development of a stricture (OR 0.40, CI 0.21-0.75, P=0.005 and OR 0.30, CI 0.11-0.79, $P=0.015$, respectively). Development of a bile leak appeared to be the only independent negative risk factor for the development of a stricture $(P=0.001)$. Donor age, recipient age, use of a Roux limb, and use of an internal stent were not independent risk factors for development of a stricture. However, when a bile leak was excluded from the multivariate analysis, use of an internal stent emerged as being protective (OR 0.32, CI 0.10-0.98, $P=0.046$ ), whereas the other covariates continued to be independent risk factors.

\section{DISCUSSION}

Our study on MELD era liver transplants identifies several risk factors for biliary complications. We differentiate factors that influence the development of anastomotic leaks from those associated with anastomotic strictures and distinguish the influence of donor, recipient, and technical factors on these separate biliary complications. Recipient age, recipient MELD score, donor age, and warm ischemia time increased the risk of bile leak in the univariate analysis, and use of a Roux limb or an internal stent decreased the risk of a bile leak in the univariate analysis, with warm ischemia time emerging as the only independent risk factor after multivariate analysis. MELD score and use of an internal stent demonstrated trends that did not quite reach statistical significance in the multivariate analysis for bile leaks. In contrast, donor age and the development of a bile leak were the only variables that increased the risk of anastomotic stricture in the univariate analysis. In addition, use of a transcystic tube or HTK preservation solution decreased the risk of an anastomotic stricture in the univariate analysis. Multivariate analysis showed that the development of a bile leak and use of HTK or a transcystic tube were the only negative and positive risk factors, respectively, that independently affected the rate of anastomotic stricture. Use of an internal stent trended toward being protective against a stricture in both the univariate and multivariate analyses but did not quite meet statistical significance.

The reported incidence of biliary complications in historical pre-MELD series is $10 \%-30 \% .^{1-5}$ Although attempts have been made to understand the risk factors involved and to study techniques of biliary reconstruction, ${ }^{1}$ these complications have remained high. Furthermore, expanded use of donor organs, institution of MELD, changes in viral prophylaxis, changes in immunosuppression, and changing procurement techniques have all occurred over the last 3-5 years. ${ }^{15}$ The impact of this changing landscape on the rate of anastomotic biliary complications and identification of contemporary risk factors was the purpose of the present study. Although historical studies indicate that cytomegalovirus infection, ${ }^{19}$ hepatic artery thrombosis or stenosis, ${ }^{10,20}$ and ABO incompatibility ${ }^{21}$ all increase the biliary complication rate, these factors are relatively minimized in contemporary series as viral prophylaxis has improved and events such as ABO incompatibility and hepatic artery thrombosis are infrequent events. ${ }^{15}$ Additionally, many of these studies evaluated intrahepatic biliary strictures rather than anastomotic complications.

Some progress has been made recently in understanding technical factors that may influence the development of anastomotic biliary complications. Recently, a randomized, controlled, multicenter trial ${ }^{9}$ has demonstrated that the use of a T-tube is associated with a higher rate of biliary complications (33.3\% versus $15.5 \%$ ) mainly secondary to a higher bile leak rate. T-tubes were not used in the current study; instead, a transcystic tube/stent was used in some patients (16\%) and was associated with a lower rate of stricture formation. It should be noted that variable practices and variable indications for use of a transcystic tube/stent may confound some of these findings in this retrospective study. Additionally, there may be some selection bias with a transcystic tube as all of these underwent cholangiography to screen for a possible leak. Although living donor liver transplantation has been associated 
with a higher biliary complication rate, ${ }^{6,8}$ some recent analyses of technical variables have provided some insight. A retrospective study by Kasahara et al. ${ }^{12}$ in living donor liver transplantation has shown that ductto-duct or primary anastomoses were associated with a lower bile leak rate of $4.7 \%$, but a higher stricture rate of $26.6 \%$, when compared to Roux-en-Y reconstruction, for which the rates were $12.4 \%$ and $8.3 \%$, respectively.

The current findings differ somewhat from those of studies on pre-MELD liver transplants. A previous study showed that Roux-en-Y reconstruction did not result in a different bile leak rate, whereas the stricture rate was higher. ${ }^{1}$ The finding of a lower bile leak rate with use of a Roux limb in the current study may be attributable to differences in donor age, ischemia time, and recipient illness severity as measured by MELD between the 2 eras. Unfortunately, we do not have accurately controlled pre-MELD data to examine this issue. The fact that a Roux limb appeared to lower the leak rate without a realizable difference in stricture rate could mean that a Roux limb has an inherent predisposition to stricture independent of a leak, similarly to previous studies. ${ }^{1}$ Although warm ischemia time has not been found to be a significant factor for development of anastomotic biliary complications, ${ }^{6,12}$ warm ischemia time was clearly an independent risk factor for deceased donor transplants in the current study. Use of a stent is clearly an unsettled issue as these same studies have had some success in decreasing the rate of stricture. ${ }^{12}$ This is somewhat in concordance with the present study in that use of a stent was protective against leaks in the univariate analysis and trended toward being significant in the multivariate analysis for being protective against both leaks and strictures. When bile leaks were excluded from the multivariate analysis for stricture, a stent was independently protective against a stricture. However, insufficient sample size limits the ability to make firm recommendations about stenting. The issue of selection bias in terms of Roux reconstruction or stenting may certainly exist in this retrospective study. Clearly, a prospective trial may help to further establish the risks and benefits of biliary stents.

MELD score was associated with a higher bile leak rate in univariate analysis but did not quite meet significance in the multivariate analysis. Another study ${ }^{6}$ had implicated certain MELD components (bilirubin level and international normalized ratio) to be risk factors for biliary complications. However, MELD, when analyzed as a single variable, was not found to be significant. In this study, recipient age was not found to be significant, whereas we found recipient age and donor age to be significant risks for bile leaks in the univariate analysis. Cold ischemia time has been implicated to cause a higher biliary complication rate; however, most of this effect has been primarily associated with intrahepatic strictures. ${ }^{22,23}$ We did not find an association of cold ischemia time with anastomotic strictures. Whether these differences in risk factors are related to changing policy trends, because of the multifactorial nature of these complications, is uncertain. Clearly, some of these changing trends have had effects on overall patient and allograft survival. ${ }^{15}$

HTK is currently widely used as a preservation solution secondary to its lower cost and acceptable graft survival outcomes. ${ }^{24}$ Smaller studies have also implicated that use of HTK may result in a lower rate of biliary complications than UW solution, ${ }^{25,26}$ which is possibly related to lower viscosity. Our present study supports this contention in that HTK was independently protective against anastomotic biliary stricture. Therefore, use of HTK may serve to limit the development of anastomotic biliary stricture, although this was not tested in a randomized fashion. Certainly, one cannot exclude a potential era effect in this retrospective study.

Our study certainly has limitations with respect to its retrospective nature and limited sample size in certain variables. As discussed earlier, sample size was limited with respect to use of stents but also with respect to some of the recipient diagnoses. We found no direct influence of diagnosis or transplant indication on the incidence of anastomotic biliary complications, although those recipients with autoimmune hepatitis appeared to have a higher rate of anastomotic stricture, whereas those with biliary atresia appeared to have a lower rate. Recent literature for PSC has failed to show an increased rate of anastomotic stricture, whereas the rates of intrahepatic strictures are clearly increased. ${ }^{27}$ However, use of Roux-en-Y reconstruction has been determined to be superior in PSC patients with a decreased stricture rate. ${ }^{13}$ Likewise, autoimmune hepatitis recipients undergoing transplantation have been shown to have higher rejection rates and intrahepatic stricture rates, with no clear evidence at this time of higher anastomotic complication rates. ${ }^{28}$

This contemporary study identifies several risk factors for anastomotic bile leaks and anastomotic strictures among MELD era liver transplants. Recipient age, donor age, MELD score, and warm ischemia time appear to have a greater impact on leak rates. Use of a stent or a Roux limb appeared to be protective against leaks, whereas other technical factors such as use of HTK and use of a transcystic tube/stent appeared to be protective against anastomotic strictures. Development of a bile leak was the largest risk factor for the development of an anastomotic stricture. The results of the current study make an argument that donor, recipient, and technical factors can differentially contribute to the risk of anastomotic biliary complications. However, warm ischemia time, HTK preservation solution, and the use of an internal stent emerged as being most important on the basis of the multivariate analysis. Technical factors, such as the decision to use a stent or Roux limb reconstruction, in the context of higher donor and recipient risk combinations are certainly worthy of additional prospective study. Although the present study is retrospective, the routine use of HTK does not appear to increase the risk of anastomotic biliary complications and may serve to lower that risk on the basis of the present data. 


\section{REFERENCES}

1. Greif F, Bronsther OL, Van Thiel DH, Casavilla A, Iwatsuki $\mathrm{S}$, Tzakis A, et al. The incidence, timing, and management of biliary tract complications after orthotopic liver transplantation. Ann Surg 1994;219:40-45.

2. Lerut J, Gordon RD, Iwatsuki S, Esquivel CO, Todo S, Tzakis A, et al. Biliary tract complications in human orthotopic liver transplantation. Transplantation 1987;43: 47-51.

3. Sutcliffe R, Maguire D, Mroz A, Portmann B, O’Grady J, Bowles M, et al. Bile duct stricture after adult liver transplantation: a role for biliary reconstructive surgery? Liver Transpl 2004;10:928-934.

4. Stratta RJ, Wood RP, Langnas AN, Hollins RR, Bruder KJ, Donovan JP, et al. Diagnosis and treatment of biliary tract complications after orthotopic liver transplantation. Surgery 1989; 106:675-683.

5. Chardot C, Candinas D, Miza D, Gunson B, Davison S, Murphy MS, et al. Biliary complications after paediatric liver transplantation: Birmingham's experience. Transpl Int 1995;8:133-140.

6. Qian YB, Liu CL, Lo CM, Fan ST. Risk factors for biliary complications after liver transplantation. Arch Surg 2004; 139:1101-1105.

7. Englesbe MJ, Dimick J, Mathur A, Ads Y, Welling TH, Pelletier SJ, et al. Who pays for biliary complications following liver transplant? A business case for quality improvement. Am J Transplant 2006;6:2978-2982.

8. Olthoff KM, Merion RM, Ghobrial RM, Abecassis MM, Fair $\mathrm{JH}$, Fisher RA, et al. Outcomes of 385 adult-to-adult living donor liver transplant recipients: a report from the A2ALL consortium. Ann Surg 2005;242:314-325.

9. Scatton O, Meunier B, Cherqui D, Boillot O, Sauvanet A, Boudjema K, et al. Randomized trial of choledochocholedochostomy with or without a T tube in orthotopic liver transplantation. Ann Surg 2001;233:432-437.

10. Nishida S, Kato T, Levi D, Naveen M, Thierry B, Vianna R, et al. Effect of protocol doppler ultrasonography and urgent revascularization on early hepatic artery thrombosis after pediatric liver transplantation. Arch Surg 2002;137: 1279-1283.

11. O'Connor TP, Lewis WD, Jenkins RL. Biliary tract complications after liver transplantation. Arch Surg 1995;130: 312-317.

12. Kashara M, Egawa H, Takada Y, Oike F, Sakamoto S, Kiuchi T, et al. Biliary reconstruction in right lobe livingdonor liver transplantation: comparison of different techniques in 321 recipients. Ann Surg 2006;243:559-566.

13. Welsh FK, Wigmore SJ. Roux-en-Y choledochojejunostomy is the method of choice for biliary reconstruction in liver transplantation for primary sclerosing cholangitis. Transplantation 2004;77:602-604.

14. Ishiko $T$, Egawa $H$, Kashara $M$, Nakamura $T$, Oike $F$, Kaihara S, et al. Duct-to-duct biliary reconstruction in living donor liver transplantation utilizing right lobe graft. Ann Surg 2002;236:235-240.

15. Shiffman ML, Saab S, Feng S, Abecassis MI, Tzakis AG,
Goodrich NP, et al. Liver and intestine transplantation in the United States, 1995-2004. Am J Transplant 2006;6: 1170-1187.

16. Merion RM, Schaubel DE, Dykstra DM, Freeman RB, Port FK, Wolfe RA. The survival benefit of liver transplantation. Am J Transplant 2005;5:307-313.

17. Campbell MS, Kotlyar DS, Brensinger CM, Lewis JD, Shetty K, Bloom RD, et al. Renal function after orthotopic liver transplantation is predicted by duration of pretransplantation creatinine elevation. Liver Transpl 2005;11: 1048-1055.

18. Pelletier SJ, Vanderwall K, Debroy MA, Englesbe MJ, Sung RS, Magee JC, et al. Preliminary analysis of early outcomes of a prospective, randomized trial of complete steroid avoidance in liver transplantation. Transplant Proc 2005;37:1214-1216.

19. Halme L, Hockerstedt K, Lautenschlager I. Cytomegalovirus infection and development of biliary complications after liver transplantation. Transplantation 2003;75: 1853-1958.

20. Abbasoglu O, Levy MF, Vodapally MS, Goldstein RM, Husberg BS, Gonwa TA, et al. Hepatic artery stenosis after liver transplantation: incidence, presentation, treatment and long-term outcome. Transplantation 1997;63:250-255.

21. Sanchez-Urdazpal L, Batts KP, Gores GJ, Moore SB, Sterioff $\mathrm{S}$, Wiesner $\mathrm{RH}$, et al. Increased bile duct complications in liver transplantation across the ABO barrier. Ann Surg 1993;218:152-158.

22. Colonna JO, Shaked A, Gomes AS, Colquhoun SD, Jurim $\mathrm{O}$, McDiarmid SV, et al. Biliary strictures complicating liver transplantation: incidence, pathogenesis, management, and outcome. Ann Surg 1992;216:344-350.

23. Sankary HN, McChesney L, Hart M, Foster P, Williams J. Identification of donor and recipient risk factors associated with nonanastomotic biliary strictures in human hepatic allografts. Transplant Proc 1993;25:1964-1967.

24. Mangus RS, Tector AJ, Agarwal A, Vianna R, Murdock P, Fridell JA. Comparison of histidine-tryptophan-ketoglutarate solution (HTK) and University of Wisconsin solution (UW) in adult liver transplantation. Liver Transpl 2006; 12 : 226-230.

25. Canelo R, Hakim NS, Ringe B. Experience with hystidine tryptophan ketoglutarate versus University of Wisconsin preservation solutions in transplantation. Int Surg 2003; 88:145-151.

26. Erhard J, Lange R, Scherer R, Kox WJ, Bretschneider HJ, Gebhard MM, et al. Comparison of histidine-tryptophanketoglutarate (HTK) solution versus University of Wisconsin (UW) solution for organ preservation in human liver transplantation. A prospective, randomized study. Transpl Int 1994;7:177-181.

27. Bjoro K, Brandsneter B, Foss A, Schrumpf E. Liver transplantation in primary sclerosing cholangitis. Semin Liver Dis 2006;26:69-79.

28. Guichelaar MMJ, Benson JT, Malinchoc M, Krom RA, Wiesner RH, Charlton MR. Risk factors for and clinical course of non-anastomotic biliary strictures after liver transplantation. Am J Transplant 2003;3:885-890. 